Génét. Sél. Evol., 1988, 20 (4), 425-434

\title{
Relationship between density- and frequency-dependent effects in two strains of Drosophila melanogaster
}

\author{
V. MOLINA, F. GONZÁLEZ-CANDELAS and J.-L. MÉNSUA \\ Universidad de Valencia, Faculdad de Ciencias Biológicas, \\ Departamento de Genética, 46100 Burjassot (Valencia), Spain
}

\begin{abstract}
Summary
Larval to adult viability was measured for two strains of Drosophila melanogaster: a wild strain (Oregon-R) and an eye colour mutant strain (cardinal). Five different densities and six genotypic frequencies were used. The results show that both strains present frequency- and density-dependent effects, but the viability of the cardinal strain always increases when its genotype frequency increases at all densities, whereas the viability of the Oregon strain behaves in a different way at low densities (where it is frequency-independent) than at higher ones (where it shows negative frequency-dependent effects).
\end{abstract}

Key words: frequency-dependence, density-dependence, larval competition, Drosophila melanogaster.

\section{Résumé}

\section{Relations entre les effets densité-dépendants et fréquence-dépendants} dans deux souches de Drosophila melanogaster

La viabilité entre les stades larvaire et adulte a été mesurée dans deux lignées de Drosophila melanogaster : une lignée sauvage (Orégon-R) et une lignée mutante pour la couleur de l'œil (cardinal). Cinq densités différentes et six fréquences génotypiques ont été utilisées. Les résultats mettent en évidence des effets densité-dépendants et fréquence-dépendants dans les deux lignées, mais la viabilité de la lignée cardinal augmente avec la fréquence de ce génotype pour toutes les densités, alors que la viabilité de la lignée Orégon se comporte différemment aux basses densités (où il n'y a pas de fréquence-dépendance) et aux densités plus élevées (où il y a des effets fréquence-dépendants négatifs). gaster.

Mots clés : fréquence-dépendance, densité-dépendance, compétition larvaire, Drosophila melano- 


\section{Introduction}

The work of WRIGHT \& DobZhansky (1946) on polymorphisms of chromosomal inversions of Drosophila pseudoobscura was among the first to raise the question of the existence of frequency-dependent selection. Classical works that have shown that the fitness of a certain genotype is affected by its own frequency in the population are, among others, the studies of Teissier (1954), Spiess (1957), Polivanov \& Anderson (1969) and Mourao et al. (1972).

There are different hypotheses about frequency-dependent selection, such as genetic facilitation (Lewontin, 1955), sexual selection (PetiT, 1951), larval competition for food (LEwONTIN, 1955), etc.

Two mechanisms have been proposed to explain the variation of larval viability in relation to frequencies in Drosophila. The first is based on the qualitative modification of the medium and the differential response of genotype with respect to this modification (Huang et al., 1971; Kojima \& Huang, 1972). The second is based on the quantitative variation of the culture medium (DE JoNG, 1976). This variation is produced by the presence of genotypes which need equal or different minimum amounts of food to reach pupation, and with equal or different probabilities of taking particles of food.

On the other hand, TURNER \& Williamson (1968) observed that changes in the selective value related to the density of a certain phenotype can produce, simultaneously, a relation between selective value and frequency. However, density-dependent selection is a field that arouses controversy, since some authors indicate that many of the experiments mostly on population dynamics, cannot differentiate between frequency-dependent and density-dependent selection. Debenedictis (1977) pointed out that frequency dependence may be questionable in cases when interaction between frequency and density is present. Tosic \& Ayala (1981) affirm that the term " frequency-dependent selection » already connotes that the selection is affected by density.

In this work we attempt to clarify the relationship between frequency- and densitydependent selection, with respect to larval viability, by means of an experimental method based on larval competition for food.

\section{Material and methods}

\section{A. Strains and culture conditions}

Two strains of Drosophila melanogaster were used: a wild strain, Oregon- $\mathrm{R}$ (isogenic) and an eye colour mutant strain, cardinal $(c d, 3: 75.7)$. The strains were kept in $150 \mathrm{ml}$ bottles containing $30 \mathrm{ml}$ of boiled yeast food (water, $1 \%$ agar, $10 \%$ sugar, $0.5 \%$ salt and $10 \%$ brewer's yeast). Stocking and experiments were carried out at $25 \pm 1{ }^{\circ} \mathrm{C}$ with $60 \pm 5 \%$ relative humidity and constant lighting. 
Eggs were laid on watch glasses with a gel layer composed of agar dissolved in water, ethanol and acetic acid. The watch glasses containing the eggs were kept in Petri dishes at $25 \pm 1{ }^{\circ} \mathrm{C}$ between 18 and 24 hours. The newly hatched larvae ( \pm 2 hours old) were seeded in the corresponding vials.

The genetic compositions used in the di-cultures were : $0.96 / 0.04,0.84 / 0.16,0.64 /$ $0.36,0.36 / 0.64,0.16 / 0.84$ and $0.04 / 0.96$. Two kinds of vials were used : large vials $(7 \times 1.3 \mathrm{~cm})$ and small vials $(5 \times 0.8 \mathrm{~cm})$ with three different amounts of food, 0.50 , 0.75 and $1.00 \mathrm{ml}(0.1 \mathrm{~g}$ of yeast per $1 \mathrm{ml}$ of medium, approximately). Different numbers of larvae were seeded: $25,50,100,150$ and 200 . A total of 180 $(6 \times 2 \times 3 \times 5)$ different experiments were performed. In each case several repetitions were made, ranging from 4 to 12 .

The emerged adults were counted daily, according to their genotypes. We calculated larva to adult viability, the fitness component studied, as $n_{0} / n_{i}$, being $n_{i}$ the number of seeded larvae and $n_{0}$ the number of emerged adults.

\section{B. Statistical procedures}

The data were suitably represented in contingency tables (FIENBERG, 1970).

Three statistical analyses (SOKAL \& ROHLF, 1981) were made after angular transformation of the viability data :

- a five-way variance analysis (genotypic frequency, larval density, amount of food, type of vial and genotype);

- analysis of variance combined with linear regression for the angular transformation of viabilities versus genotypic frequency at every larval density. This analysis enables us to test whether each slope is significantly different from 0 for every density and for each strain. The $F$-tests are compared with an $F_{1, n}$ table, where the degrees of freedom in the numerator correspond to the degree of the fitted equation, and the degrees of freedom in the denominator are the product of the number of classes minus 1 and the number of repetitions;

- a test for comparison of regression lines. This analysis enables us to test whether the five groups (five densities) were sampled or not from populations of equal slopes. The analysis is made for each strain separately.

\section{Results}

Table 1 shows the variance analysis for the angular transformation of viabilities. In this analysis, the five individual factors (genotype frequency, larval density, amount of food, type of vial and genotype) are significant at least at the 0.05 level. It is worth mentioning that all the interactions in which the genotype frequency appears are not significant, except the frequency $(1) \times$ genotype $(5)$ interaction (table 1$)$. This interaction is significant because the Oregon strain presents a negative frequency-dependent selection while the cardinal strain presents a positive one. 
TABLE 1

Five-way variance analysis for the angular transformation of viabilities

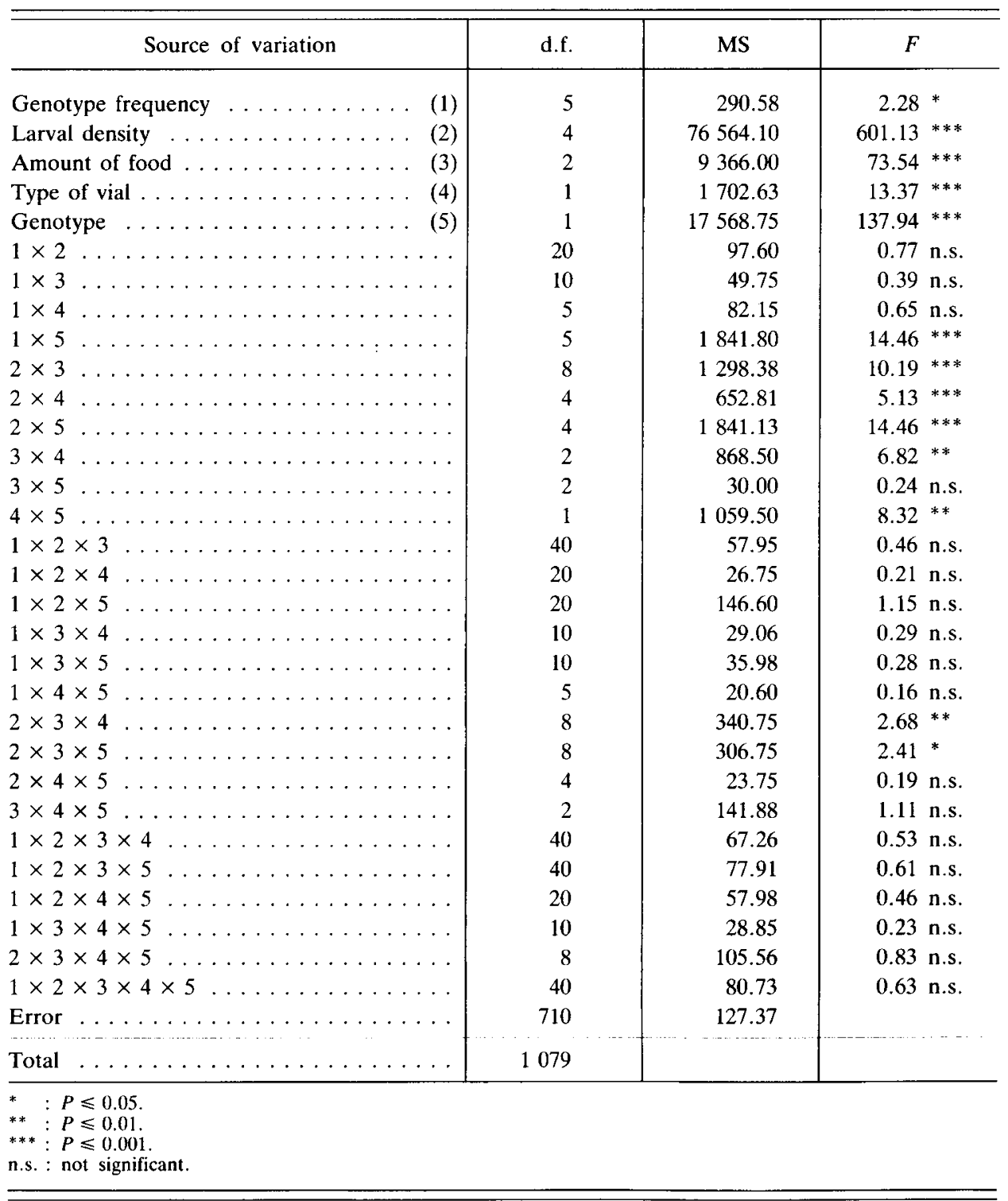

All the interactions in which frequency and density appear simultaneously with other factors are not significant. So, for each combination frequency $\times$ density we have six data ( 3 from the number of vials $\times 2$ from the different amounts of food). These data are considered as repetitions in the following analyses. 
Figure 1 shows the regressions of the angular transformation of viabilities versus genotype frequency at different densities. These data were taken from the frequency$\times$ density $\times$ genotype contingency tables.

The variance analysis combined with linear regression (table 2) showed that all the slopes were significantly different from zero except for the two regressions of the Oregon strain at initial larval densities of 25 and 50, respectively.

TABLE 2

F-tests from variance analysis combined with regression for the angular transformation of viabilities. The test checks whether each slope (b) is significantly different from 0

\begin{tabular}{c|r|r|r|r}
\hline \hline Strain & Density & $a$ & \multicolumn{1}{c|}{$b$} & $F(1,30)$ \\
\hline \multirow{2}{*}{ Oregon $\ldots \ldots \ldots \ldots$} & 25 & 56.77 & 1.82 & 4.24 n.s. \\
& 50 & 50.57 & 1.01 & 0.05 n.s. \\
& 100 & 40.20 & -9.57 & $19.09^{* * *}$ \\
& 150 & 35.43 & -16.03 & $44.82^{* * *}$ \\
& 200 & 21.01 & -6.68 & $19.62^{* * *}$ \\
& 25 & 53.14 & 7.12 & $10.04^{* *}$ \\
& 50 & 42.19 & 10.16 & $13.87^{* * *}$ \\
& 100 & 17.79 & 13.05 & $17.86^{* * *}$ \\
& 150 & 4.84 & 13.60 & $93.46^{* * *}$ \\
& 200 & 4.19 & 9.83 & $28.58^{* * *}$ \\
\hline
\end{tabular}

** : $P \leqslant 0.01$.

***: $P \leqslant 0.001$.

n.s. : not significant.

$a$ is the intercept at the origin.

$b$ is the slope of the regression.

In table 3 a variance analysis to test whether the slopes of different regressions for each density can be considered equal or not is shown. As can be observed, the regression coefficient of the cardinal strain can be considered the same at all densities. The average regression coefficient is $b_{\mathrm{A}}=10.75$. In the Oregon strain, there are significant differences among regression coefficients. However, regarding only the three highest densities (where the regressions were significant) it can be observed that the slopes of viability are the same and the average regression coefficient is $b_{\mathrm{A}}=-10.83$.

\section{Discussion}

From our results, we can conclude that, with respect to viability, both strains present frequency-dependent effects and density-dependent effects (since both individual factors are significant). As frequency $\times$ density interaction is not significant, the problem suggested by Debenedictis (1977) is avoided. 


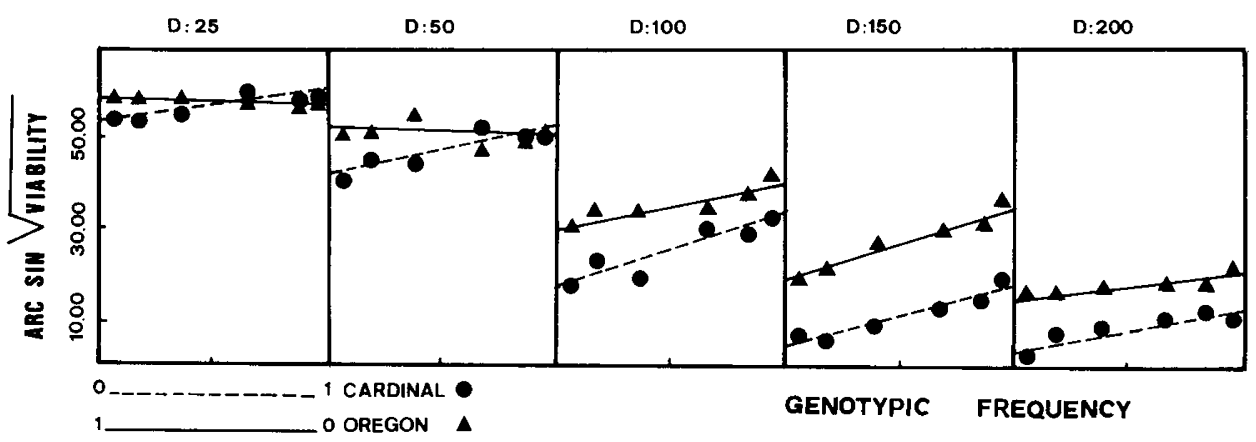

FIG. 1

Angular transformation of viability

as a linear function of genotype frequency at different densities (D).

The viability of the cardinal strain always increases when its genotype frequency increases (positive frequency-dependent selection), and this increase of viability in relation to frequency is invariably the same at all the densities. In this strain the increase in density produces a decrease in viability as a whole.

The viability of the Oregon strain behaves in a different way at low densities than at higher ones, similarly to what Tosic \& Ayala (1971) observed working with the Mdh-2 locus of Drosophila pseudoobscura. At low densities (25 and 50) there are no frequency-dependent effects, but these appear at higher ones (initial densities over 100 individuals). From this point, the selection coefficient remains constant as in the cardinal strain case.

The cardinal strain behaviour seems to be contrary to the assertion made by Tosic \& Ayala (1981) that the term "frequency-dependent selection » already connotes that the selection is affected by density ; in the range of densities studied it is observed that in the cardinal strain the variation of viability with frequency is independent of density. We will discuss this point further. In table 3 we can see that slopes for the cardinal strain are not significantly different for all the densities.

A physiological explanation, in our case, for these behaviours can be obtained from two works : Economos et al. (1982) found that $1 \mathrm{mg}$ of yeast is enough for one larva to develop. CASTRo et al. (1986) showed that the cardinal larvae excrete less uric acid (which is toxic to larvae, because it lowers viability and delays developmental time (CASTro et al., 1987)), eat significantly less food and are less resistant to biotic residues than other strains of Drosophila melanogaster. On the contrary, the normal strains, like Oregon, consume more food and excrete more biotic residues but are more resistant to them (CASTRo et al., 1987).

In our work, two ranges of densities are distinguished. The former, in which every larva has an amount of yeast equal to or greater than $1 \mathrm{mg}$, and the second, in which every larva has less than $1 \mathrm{mg}$ of yeast.

In the first case $(D=25$ and 50 ) the Oregon larvae do not present frequencydependent effects. This can be due to both having enough food to develop and a low number of larvae producing biotic residues in such an amount that they are not affected 
TABLE 3

Test for equality of slopes of viability regression lines

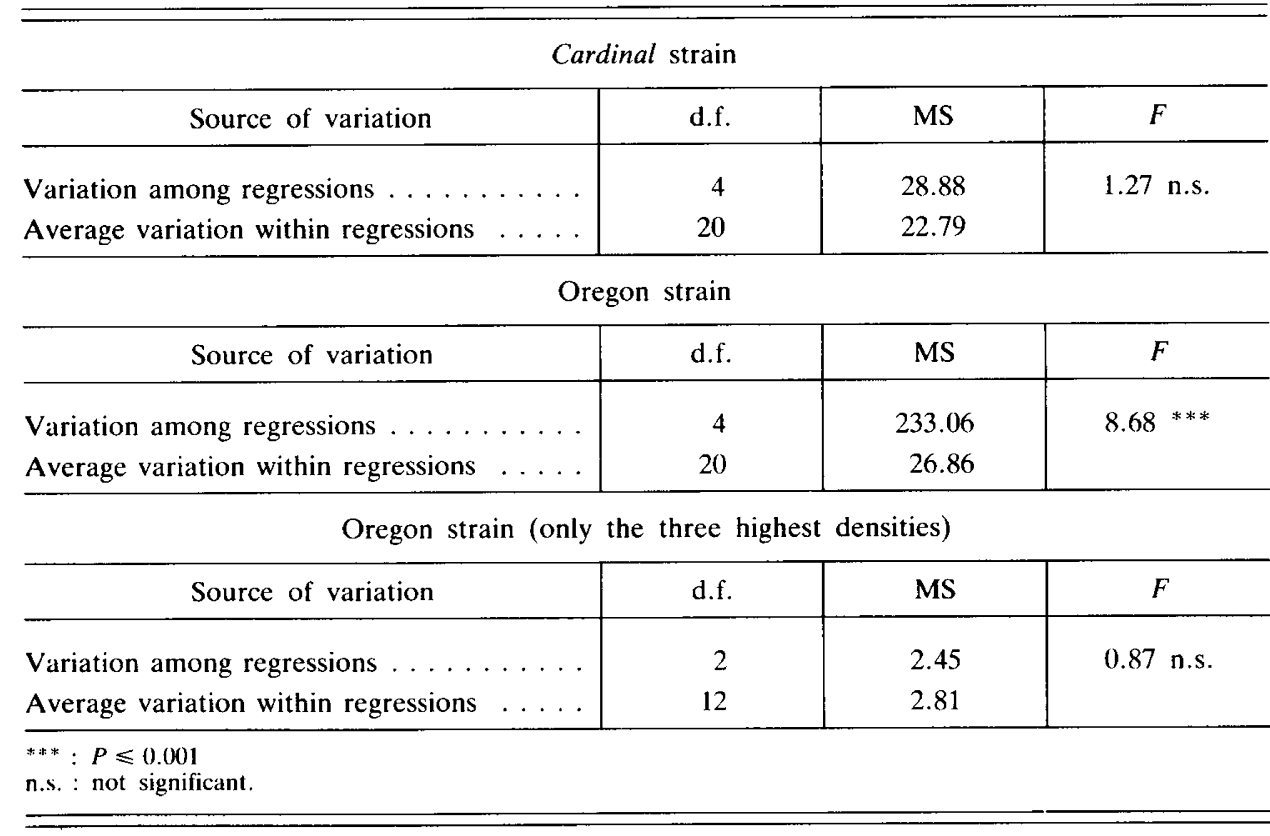

seriously. However, at these densities the cardinal larvae present positive frequencydependent effects. It can be assumed that despite the fact that there is enough yeast, as they are more sensitive to biotic residues, they are more affected when the frequency of the Oregon strain increases, because this strain excretes a higher amount of residues.

In the second case (densities higher than 50) the Oregon larvae present negative frequency-dependent selection. In this case, this may be due to the shortage of food for larvae to develop properly, and to the amount of biotic residues in the medium which is then high enough to affect larvae. The lower the frequency of the Oregon strain, the smaller is the amount of biotic residues. Furthermore larvae will have proportionnally a higher amount of food. On the contrary, the cardinal larvae will continue to present positive frequency-dependent selection. The higher their frequency the more favoured they will be, since they eat significantly less food and excrete less biotic residues than the Oregon larvae.

Therefore, the increasing number of larvae of the cardinal strain has a positive effect on themselves and on the Oregon larvae, as occurred with the larvae of the $A m y^{1}$ strain on the $A m y^{\text {nul1 }}$ strain in the work of HaJ-Ahmad \& Hickey (1982). However, the increment of Oregon larvae has a negative effect on themselves as well as on the cardinal larvae.

Therefore, in the case of larval competition for food, the factor that produces frequency-dependent effects is not directly density, but the intrinsic characteristics of each strain (amount of uric acid excreted, resistance to it, amount of food necessary to 
reach pupation, etc.). These intrinsic characteristics will affect the strain itself (intragenotypic competition) and the competitor strain (intergenotypic competition) either in a positive or a negative way. These effects will be observed from a certain density onwards but not before it (depending on the strain).

Thus, the present results lead us to think that the effect of density on frequencydependent effects is only to provoke its appearance. When these effects have appeared, although density increases, the slopes of viability versus genotype frequency (frequencydependence) are constant, whereas total viability decreases with higher densities (density-dependence). This is observed more clearly in the Oregon strain. In the range of densities studied the cardinal strain always shows frequency-dependence. This fact is due to the higher intragenotypic competition of the cardinal strain (CASTRo et al., 1985). It would be necessary to study the behaviour of this strain at lower densities, where density-dependent and frequency-independent effects could be detected, and so, the apparent contradiction with the Tosic \& Ayala (1981) assertion is explained.

According to Wallace's classification (1981), we think that frequency dependence and density independence cannot occur simultaneously. In our case there are both density and frequency dependence (except in two cases of the Oregon strain), which, according to WALLACE (1981) should be named « soft selection ». Whenever frequencydependent selection appears, it is concomitant with density dependence. But density dependence can be present without frequency dependence (Oregon strain in $D=25$ and 50).

It is possible to think that at higher densities than the ones we used, when the culture is in the chaos zone (MAY, 1975 ; HASSELL et al., 1976), the constancy of the slopes would disappear, since in this zone there is a great larval mortality and the response would be erratic (MoYA \& CASTro, 1986).

Received July 15, 1987. Accepted January 4, 1987.

\section{Acknowledgements} gació ».

Part of this work was supported by a grant of «Institució Valenciana d'Estudis i Investi-

\section{References}

Castro J.A., Moya A., Ménsua J.L., 1985. Competitive selection in mono-, di- and tri-genotype cultures of Drosophila melanogaster. Z. Zool. Syst. Evol. Forsch., 23, 214-228.

Castro J.A., Botella L.M., Ménsua J.L., 1986. Effect of conditioned media on three genotypes of Drosophila melanogaster. Arch. Insect. Biochem. Physiol., 3, 405-497.

Castro J.A., Botella L.M., MÉnsua J.L., 1987. Intra- and intergenotypic larval competition in Drosophila melanogaster : effect of larval density and biotic residues. Génét. Sél. Evol., 19 (4), 427-444. 
Debenedictis P.A., 1977. Studies in the dynamics of genetically variable populations. 1. Frequency- and density-dependent selection in experimental populations of Drosophila melanogaster. Genetics, 87, 343-356.

De Jong G., 1976. A model of competition for food. 1. Frequency-dependent viabilities. Amer. Nat., 110, 1013-1027.

Economos A.C., LiNTs C.V., LiNTs F.A., 1982. On the mechanisms of the effects of larval density and temperature on Drosophila development. In : LakovaAra S. (ed.), Advances in genetics, development, and evolution of Drosophila, 149-164, Plenum Press, New York.

Fienberg S.A., 1970. The analysis of multidimensional contingency tables. Ecology, 51, 419-433.

HaJ-Ahmad Y., Hickey D.A., 1982. A molecular explanation of frequency-dependent selection in Drosophila. Nature, 299, 350-352.

Hassell M.P., Lawton J.M.., MaY M., 1976. Patterns of dynamical behaviour in single-species populations. J. Anim. Ecol., 45, 471-486.

HuANG S.L., Sing M., Kojima K.I., 1971. A study of frequency-dependent selection observed in esterase-6 locus of Drosophila melanogaster using a conditioned media method. Genetics, 68, 97-104.

Kojima K.I., Huang S.L., 1972. Effects of population density on the frequency-dependent selection in the esterase-6 locus of Drosophila melanogaster. Evolution, 26, 313-321.

Lewontin R.C., 1955. The effects of population density and composition on viability in Drosophila melanogaster. Evolution, 9, 27-41.

MAY R.M., 1975. Some notes on estimating the competition matrix alpha. Ecology, 56, 737-741.

Mourao C.A., Ayala F.J., Anderson W.W., 1972, Darwinian fitness and adaptedness in experimental populations of Drosophila willistoni. Genetics, 43, 552-574.

MoyA A., CASTRo J.A., 1986. Larval competition in Drosophila melanogaster : the model of the bands of density. Oikos, 47, 280-286.

Perrt C., 1951. Le rôle de l'isolement sexuel dans l'évolution des populations de Drosophila melanogaster. Bull. Biol. Fr. Bel., 85, 392-418.

Polivanov S., Anderson W.W., 1969. Selection in experimental populations. 2. Components of selection and their fluctuations in two populations of Drosophila melanogaster. Genetics, 63, 919-932.

SoKal R.R., Rohlf F.J., 1981. Biometry. 776 p., Freeman, San Francisco.

SPIESs E.B., 1957. Relation between frequencies and adaptative values of chromosome arrangements in Drosophila persimilis. Evolution, 11, 84-93.

Teissier G., 1954. Conditions d'équilibre d'un couple d'allèles et supériorité des hétérozygotes. C.R. Acad. Sci. Paris, 238, 621-623.

Tosic M., Ayala F.J., 1981. Density and frequency dependent selection at Mdh-2 locus in Drosophila pseudoobscura. Genetics, 97, 679-701.

Turner J.R.G., Williamson M.H., 1968. Population size, natural selection, and the genetic load. Nature, 228, 700.

WAllace B., 1981. Hard and soft selection. In : Basic population genetics, 409-439, Columbia University Press, New York.

Wright S., Dobzhansky Th., 1946. The genetics of natural populations. 12. Experimental reproduction of some changes caused by natural selection in certain populations of Drosophila pseudoobscura. Genetics, 31, 125-156. 